\title{
Simulation of core turbulence measurement in Tore Supra ohmic regimes
}

S. Hacquin, J. Citrin, H. Arnichand, R. Sabot, C. Bourdelle, X. Garbet, A. Krämer-Flecken, and

Citation: Physics of Plasmas 23, 092303 (2016); doi: 10.1063/1.4962762

View online: http://dx.doi.org/10.1063/1.4962762

View Table of Contents: http://aip.scitation.org/toc/php/23/9

Published by the American Institute of Physics

\section{Articles you may be interested in}

Experimental observation of turbulence transition and a critical gradient threshold for trapped electron mode in tokamak plasmas

Physics of Plasmas 23, 060702 (2016); 10.1063/1.4953427

Zonal-flow dynamics from a phase-space perspective

Physics of Plasmas 23, 122304 (2016); 10.1063/1.4971813

Zonal flow generation in parallel flow shear driven turbulence

Physics of Plasmas 24, 032304 (2017); 10.1063/1.4978485

Multi-channel transport experiments at Alcator C-Mod and comparison with gyrokinetic simulations

Physics of Plasmas 20, 056106 (2013); 10.1063/1.4803089

Collisionless damping of zonal flows in helical systems

Physics of Plasmas 13, 012501 (2006); 10.1063/1.2149311

Quantitative comparison of electron temperature fluctuations to nonlinear gyrokinetic simulations in C-Mod Ohmic L-mode discharges

Physics of Plasmas 23, 042303 (2016); 10.1063/1.4945620

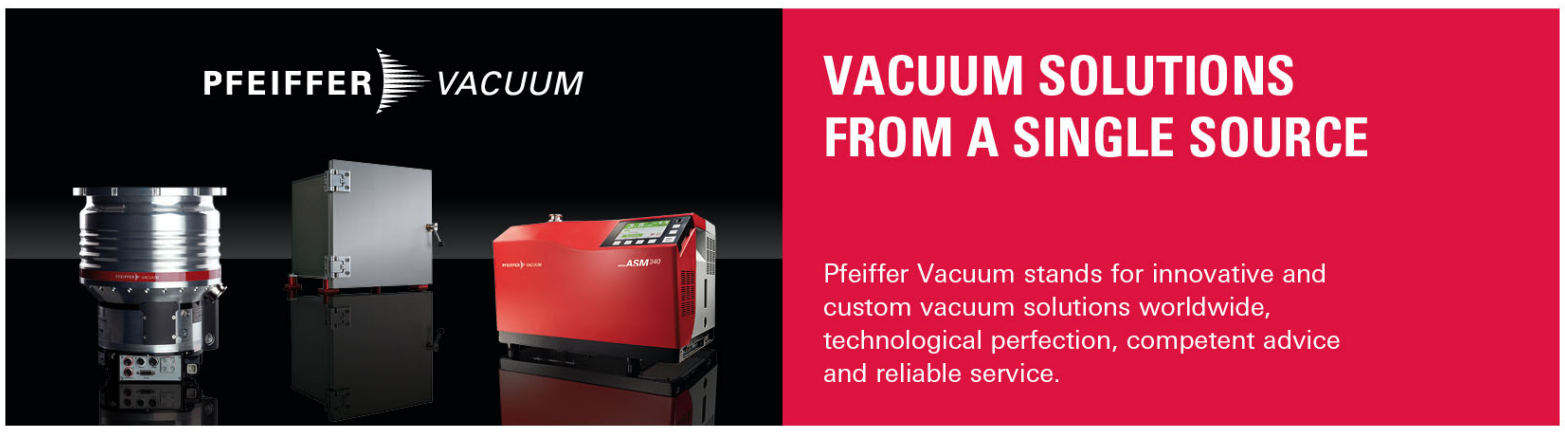




\title{
Simulation of core turbulence measurement in Tore Supra ohmic regimes
}

\author{
S. Hacquin, ${ }^{1, \text { a) }}$ J. Citrin,,${ }^{1,2}$ H. Arnichand,${ }^{1}$ R. Sabot, ${ }^{1}$ C. Bourdelle, ${ }^{1}$ X. Garbet, ${ }^{1}$ \\ A. Krämer-Flecken, ${ }^{3}$ and Tore Supra Team ${ }^{1}$ \\ ${ }^{1}$ CEA, IRFM, F-13108 Saint-Paul-lez-Durance, France \\ ${ }^{2}$ FOM Institute DIFFER-Dutch Institute for Fundamental Energy Research, PO Box 6336, \\ 5600 HH Eindhoven, The Netherlands \\ ${ }^{3}$ Institut für Energie- und Klimaforschung/Plasmaphysik, Forschungszentrum Jülich GmbH, D-52425 Jülich, \\ Germany
}

(Received 11 March 2016; accepted 21 August 2016; published online 16 September 2016)

This paper reports on a simulation of reflectometry measurement in Tore Supra ohmic discharges, for which the experimental observations as well as gyrokinetic non-linear computations predict a modification of turbulence spectrum between the linear (LOC) and the saturated ohmic confinement (SOC) regimes. Synthetic reflectometry simulations coupling full-wave computations with gyrokinetic data are carried out. This allows a direct comparison between the gyrokinetic non-linear predictions and experimental observations. The synthetic diagnostic results are found in a good agreement with the experimental findings; in particular, they reproduce well the quasi-coherent peak in the fluctuation spectrum of LOC regimes dominated by a trapped electron mode turbulence. It is also shown that such synthetic tools are valuable for (i) an enhanced interpretation of the reflectometry measurement (for instance, through the investigation of the 2D effects) and (ii) a better understanding of the turbulence properties (for instance, via the analysis of its poloidal asymmetry). Published by AIP Publishing.

[http://dx.doi.org/10.1063/1.4962762]

\section{INTRODUCTION}

Turbulence plays a key role in an anomalous transport that degrades the energy confinement time in the magnetic fusion devices, ${ }^{1}$ making the study of turbulence properties of a prime interest. Turbulence is commonly attributed to the micro-instabilities, which can be of various natures, with the ion temperature gradient (ITG) and trapped electron modes (TEM) being the dominant ones in the plasma core region. ${ }^{2}$ As ITG and TEM have been found to produce different effects on the plasma behavior, for instance, on the intrinsic toroidal rotation ${ }^{3}$ or the particle transport, ${ }^{4}$ identifying the dominant mode is vital to investigate the turbulence mechanisms. However, discriminating ITG- and TEM-dominated regimes experimentally remains challenging, mainly due to the fact that their wavenumbers lie in the same scale $0.1<\mathrm{k}_{\perp} \rho_{\mathrm{i}}<1 .^{5}$

While modifications of fluctuation spectra due to a change of turbulence regime have been reported from different diagnostics in various machines, ${ }^{6-9}$ it was recently established that the ITG- and TEM-dominated regimes could be differentiated from the reflectometry spectra. ${ }^{10}$ In the latter work, the experimental observations were supported by the non-linear gyrokinetic simulations, which qualitatively reproduced the shape of the reflectometry spectra in the ITG and TEM regimes. A direct and more quantitative comparison of the gyrokinetic computations with the experimental results requires the use of synthetic diagnostics, the development of which has become widespread in a fusion research. ${ }^{11}$ Synthetic diagnostics are particularly relevant for an interpretation of the reflectometry data since the link between the

\footnotetext{
a) sebastien.hacquin@cea.fr
}

reflectometer signal and the density fluctuations is not always straightforward. ${ }^{12}$

This paper reports on the simulation via synthetic diagnostics of reflectometry measurements in Tore Supra ohmic discharges. It is organized as follows. In Sec. II, the characteristics of the core turbulence spectra in Tore Supra ohmic discharges, inferred from the reflectometry measurement as well as from the non-linear gyrokinetic predictions, are briefly exposed. Section III deals with the presentation of the results obtained from the synthetic reflectometry simulations. Some concluding remarks and the perspectives of this work are finally discussed in Sec. IV.

\section{TURBULENCE CHARACTERISTICS IN TORE SUPRA OHMIC DISCHARGES}

The work reported in this paper focuses on the ohmic discharges in the Tore Supra tokamak. The ohmic discharges exhibit two distinct confinement regimes, the so-called linear ohmic confinement (LOC) and saturated ohmic confinement (SOC) regimes, the transition from one to the other being achieved from a scan of either the density or the plasma current. ${ }^{13}$ As it is commonly alleged that, in the most plasma scenarios, the LOC and the SOC regimes are generally dominated by TEM and ITG, respectively, ${ }^{14-16}$ ohmic discharges are of interest to study separately these two turbulence regimes.

\section{A. Experimental observations from the reflectometry measurements}

The experimental characterization of fluctuation spectra in Tore Supra ohmic discharges has been achieved with a heterodyne $\mathrm{X}$-mode reflectometry system probing the 
equatorial mid-plane in the D-band frequency range (110-160 GHz) with two Gaussian optics lens antennas for an emission and a reception of the probing signal. ${ }^{17}$ The fluctuation spectra inferred from the reflectometry measurements usually show some distinct features in the ITG- and TEM-dominated regimes. While a broadband spectrum is typically observed in the ITG-dominated regimes, the appearance of quasi-coherent modes in the TEM-dominated regimes has been evidenced in a number of the existing fusion devices: T-10, ${ }^{18}$ TEXTOR, ${ }^{19}$ Tore Supra, ${ }^{10,13}$ JET, ${ }^{20}$ ASDEX-Upgrade, ${ }^{10}$ etc. The quasi-coherent modes associated with TEM-dominated turbulence (QC-TEM) can be identified in the frequency spectra of the reflectometry signals displaying a carrier frequency on the order of magnitude of $50-150 \mathrm{kHz}$ with a bandwidth of about $10-50 \mathrm{kHz}$. As for any drift-wave turbulence, their amplitude is ballooned in the mid-plane low-magnetic-field-side (LFS) region. ${ }^{21}$ Other properties of QC-TEM modes have been more recently highlighted: a poloidal rotation velocity shifted towards the electron diamagnetic direction ${ }^{13}$ and a long-range correlation length. $^{22}$

\section{B. Non-linear gyrokinetic simulations}

To help interpret the measurement of QC modes in Ohmic discharges, the local non-linear gyrokinetic computations were carried out with the GENE code. ${ }^{23}$ Using the main parameters of Tore Supra Ohmic discharge \#48102, the turbulence spectra were estimated for both the LOC and the SOC regimes, displaying significant differences: ${ }^{24}$ first, the spectrum peak was found in the electron diamagnetic direction for the LOC regime and in the ion diamagnetic direction for the SOC regime; second, while the spectrum displays a typical broadband structure in the case of the SOC regime, a second narrower peak reminiscent of a QC mode appears in the LOC regime. Fig. 1 shows the fitted radial profiles of density and temperature, as well as the magnetic field, of both the LOC (a) and SOC (b) phases of Tore Supra discharge \#48102. These were input into the CRONOS integrated modelling code, ${ }^{25}$ where the interpretative simulations provided the magnetic geometry and equilibrium used in the GENE non-linear simulations. Figs. 1(c) and 1(d) display the density fluctuations in the poloidal plane at an initial time (the color bar corresponding to $\delta n_{e} / n_{e}$ in \%) resulting from the GENE non-linear simulations. Figs. 2(a) and 2(b) shows the radial and time evolution of the equatorial midplane fluctuations, while Figs. 2(c) and 2(d) show the poloidal rotation velocity inferred from cross-correlation of the fluctuations at two distinct poloidal angles, respectively. In order to simulate the experimental reflectometry measurements, the density fluctuations given by GENE were used as an input in full-wave reflectometry code computations, which are presented in Sec. III.

\section{SYNTHETIC REFLECTOMETRY SIMULATIONS}

\section{A. Full-wave computations}

The reflectometry simulations reported here were completed with the full-wave computations of the Maxwell's equations in the presence of a cold plasma tensor. ${ }^{26}$ The plasma was described by the radial profiles of electron density and temperature displayed in Figs. 1(a) and 1(b), consistently with the inputs used in the GENE simulations. The electron temperature was taken into account for correction of the electron mass due to the relativistic effects. ${ }^{27} \mathrm{~A}$ radial profile inversely proportional to the major radius was considered for the magnetic field, the value of which is required for
LOC
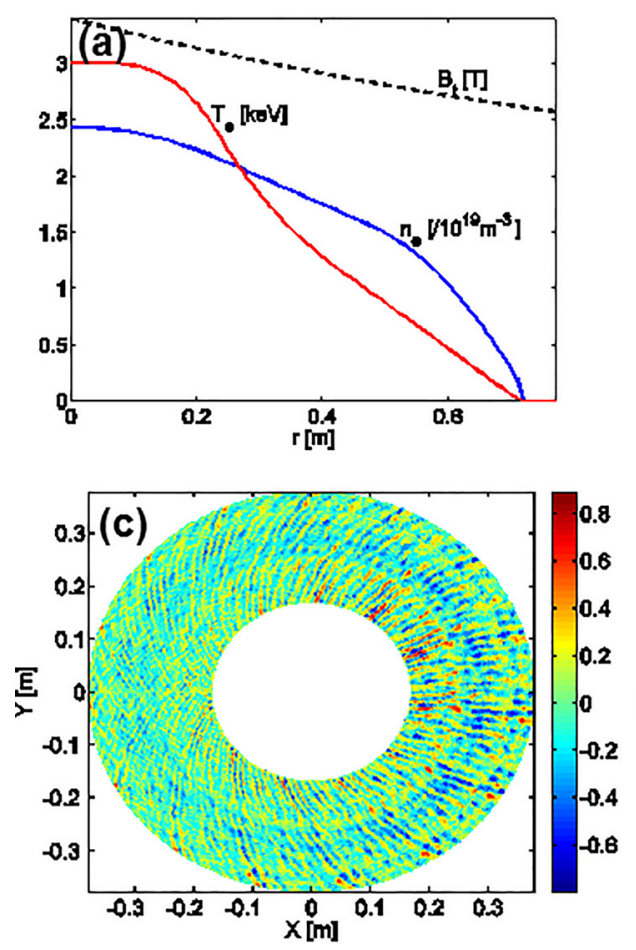

SOC
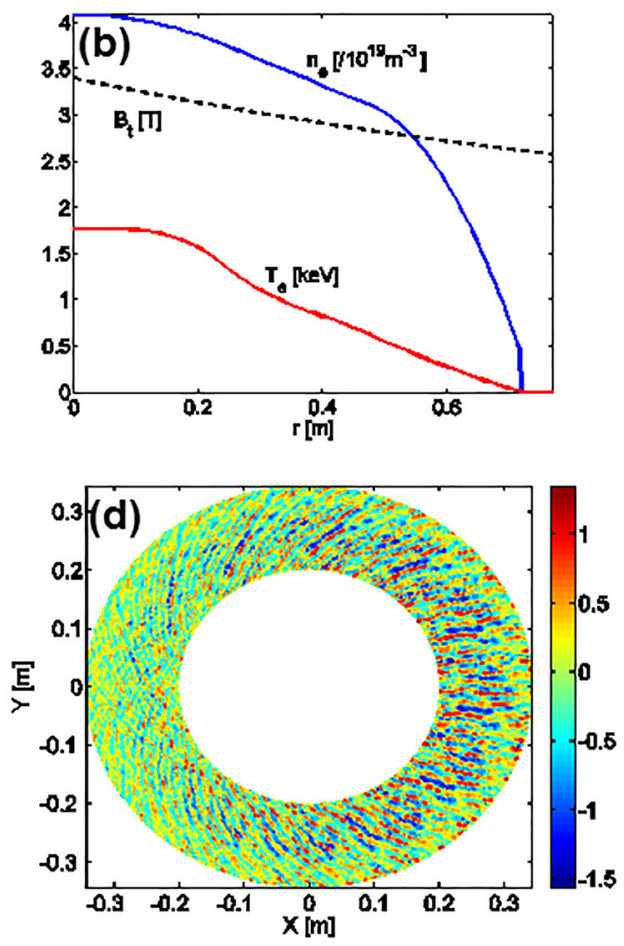

FIG. 1. GENE simulations of the Tore Supra ohmic discharge \#48102 in the LOC (left hand-side) and the SOC (right hand-side) regimes: (a) and (b) radial profiles of electron density, electron temperature, and magnetic field used as inputs; (c) and (d) density fluctuations in the poloidal section at initial time $t=0$. 
LOC
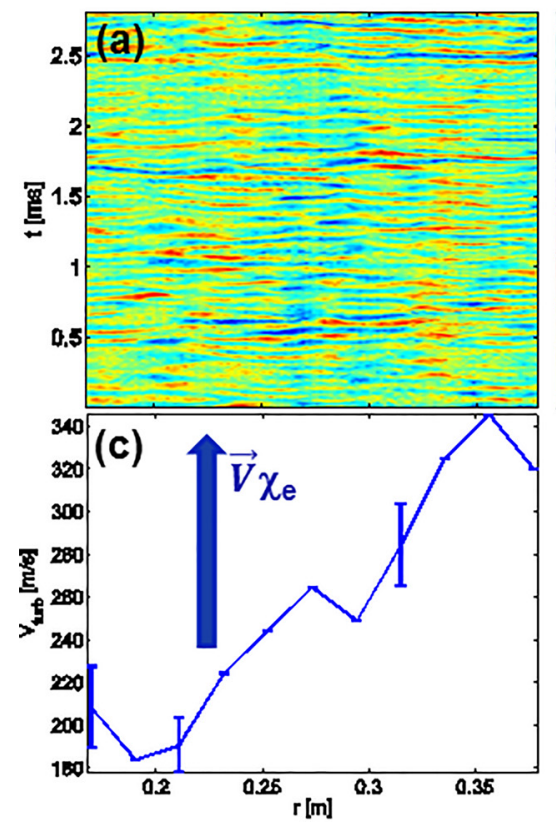

SOC
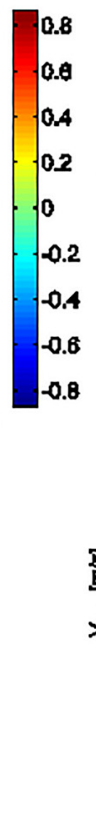

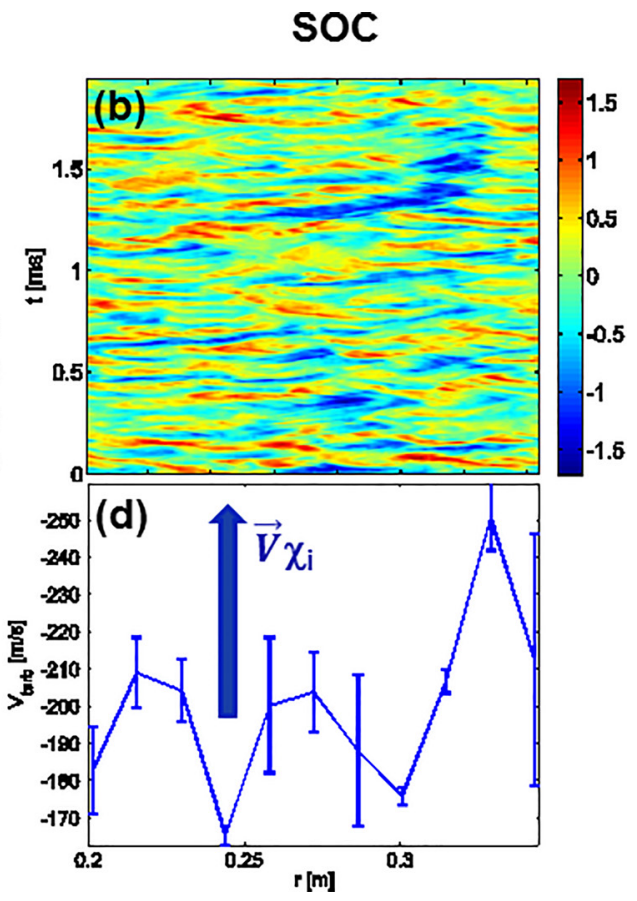

FIG. 2. GENE simulations of the Tore Supra ohmic discharge \#48102 in the LOC (left hand-side) and the SOC (right hand-side) regimes: (a) and (b) time evolution of the radial density fluctuations in the LFS mid-plane and (c) and (d) radial profiles of the fluctuation poloidal rotation velocity in the LFS mid-plane. the simulation of $\mathrm{X}$-mode reflectometry. The corresponding radial profiles for the $\mathrm{O}$-mode and the upper $\mathrm{X}$-mode cut-off frequencies are depicted in Fig. 3 for the LOC (a) and (c) and SOC regimes (b) and (d). The vertical dashed lines delimit the zone of density fluctuations computed by GENE, as set by the limits of the radial box. The horizontal dashed red lines indicate the probing frequencies that are required to probe the radial midpoint of the density fluctuation zone, namely, $41 \mathrm{GHz}$ for the $\mathrm{O}$-mode and $102 \mathrm{GHz}$ for the $\mathrm{X}$-mode in the LOC regime and $\sim 55 \mathrm{GHz}$ for the $\mathrm{O}$-mode and $\sim 112 \mathrm{GHz}$ for the $\mathrm{X}$-mode in the LOC regime. In order to reproduce the reflectometry signal, the full-wave computations are performed for each map of the density fluctuations given at the successive time steps. As done in the real experiments, the probing wave is launched from the low field magnetic side in the equatorial mid-plane.

The GENE simulations used in this work yield the time evolution of the density fluctuations in the plasma frame, independently on the contribution of the $\mathrm{E} \times \mathrm{B}$ rotation velocity to the turbulence overall movement that is seen by
LOC

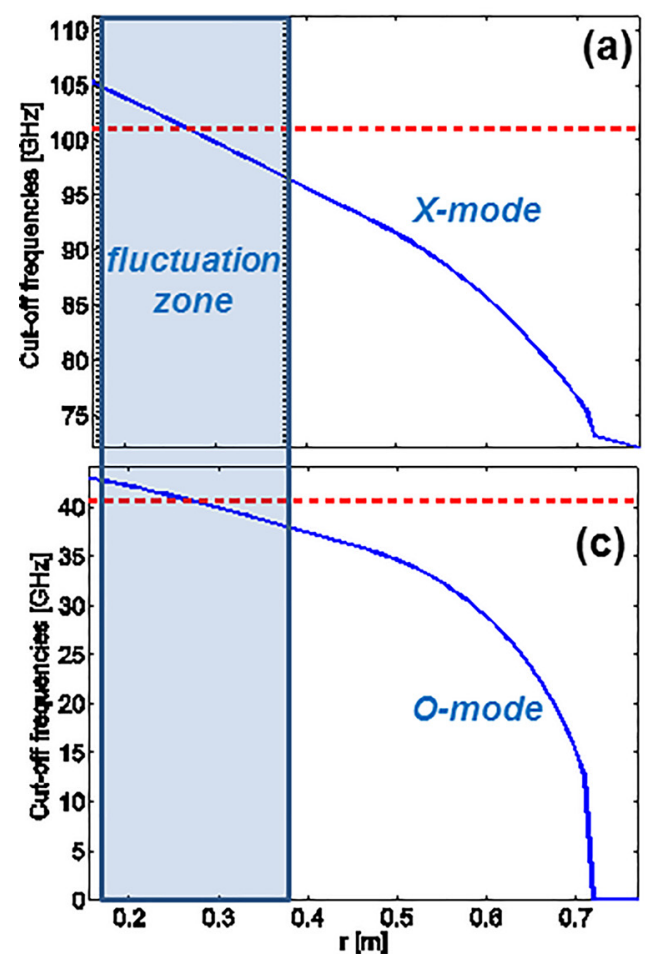

SOC

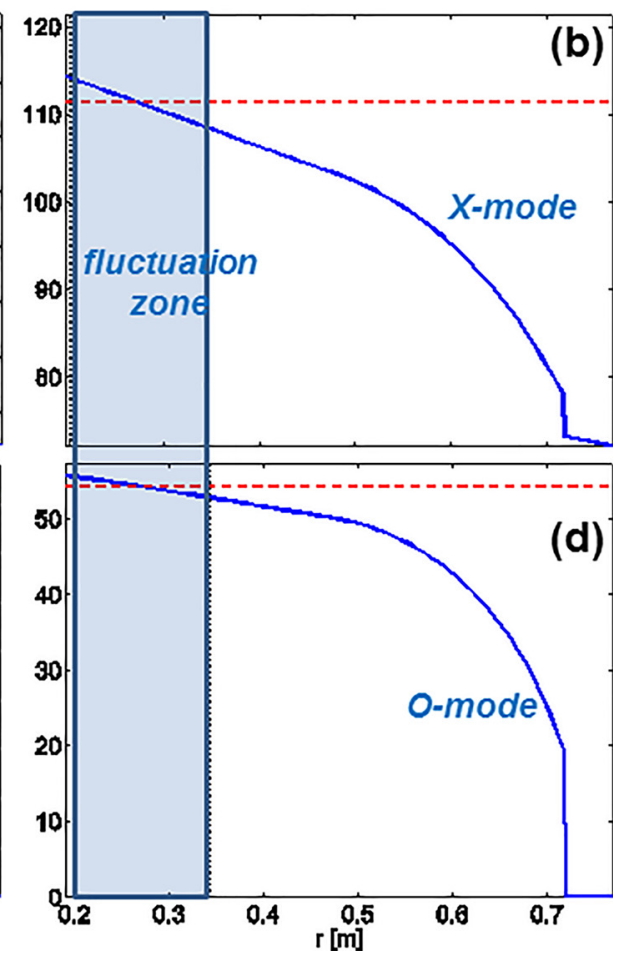

FIG. 3. Radial profiles of the cut-off frequencies for the Tore Supra ohmic discharge \#48102 in the LOC (left hand-side) and the SOC (right handside) regimes: (a) and (b) X-mode upper cut-off frequency and (c) and (d) O-mode cut-off frequency. 
the diagnostics. The $\mathrm{E} \times \mathrm{B}$ rotation velocity is usually much higher than the turbulence rotation velocity ${ }^{28}$ so that its effect on the synthetic reconstruction of the reflectometry signal is expected to be substantial. Including the $\mathrm{E} \times \mathrm{B}$ rotation velocity contribution to the reflectometry signal is not straightforward. We supposed that the turbulence was rotating poloidally as a rigid body, what was found from the GENE data as an acceptable assumption. Under this hypothesis, the sampling interval $\delta t$ for the reflectometry signal can be expressed as

$$
\delta t=\delta t_{G E N E} \times\left[\frac{V_{E \times B}}{V_{t u r b}} \pm 1\right]^{-1}
$$

where $\delta t_{G E N E}$ represents the GENE time discretization, $V_{E \times B}$ the $\mathrm{E} \times \mathrm{B}$ rotation velocity, and $V_{\text {turb }}$ the turbulence rotation velocity in the plasma frame. The sign \pm in (1) takes into account the direction of a turbulence propagation with respect to the $\mathrm{E} \times \mathrm{B}$ rotation direction. For the $\mathrm{LOC}$ regime, the sign + is used in (1) as both the $\mathrm{E} \times \mathrm{B}$ rotation, and the turbulence propagate in the electron diamagnetic direction. For the SOC regime, the turbulence propagates opposite in the ion diamagnetic direction, imposing then the use of sign - in (1). The $\mathrm{E} \times \mathrm{B}$ rotation velocity $V_{E \times B}$ in (1) was deduced from the radial electric field, which can be estimated by the thermal ripple losses in case of a strong ripple such as in Tore Supra. ${ }^{29}$ This approach to include the E $\times \mathrm{B}$ rotation contribution relies on the hypothesis that the turbulence propagates as a rigid body and is also affected by the uncertainty on $V_{\text {turb }}$ and $V_{E \times B}$ determinations, thus limiting its precision. For instance, the $V_{\text {turb }}$ changes with the radial position as shown in Figs. 2(c) and 2(d), and the value at the cut-off layer position was considered in (1), since the reflectometry signal is dominantly sensitive to this position. However, the method based on (1) enables to include the $\mathrm{E} \times \mathrm{B}$ rotation contribution and, that way, allows the reflectometry signal reconstruction as consistently as possible with the experiment.

\section{B. Comparison between O-mode and X-mode results}

In the turbulence measurements obtained in the Tore Supra ohmic discharges with a heterodyne X-mode system, the two Gaussian optics lens antennas used for the emission and reception of the probing signal lie far away (i.e., about $1 \mathrm{~m}$ ) from the plasma, so that one might expect an 1D approximation to be quite relevant to simulate such measurements. We report in this section the 1D simulations, which were carried out with a full-wave code solving the Helmholtz equation for either the $\mathrm{O}-$ mode $^{30}$ or the $\mathrm{X}-$-mode ${ }^{31}$ polarization. Such a code based on solving the Helmholtz equation relies on the matrix inversion techniques and does not require large iteration numbers of time steps to reach the stationary regime, thus having the advantage to be much faster and more accurate than full-wave codes using finite difference time domain (FD-TD) schemes. ${ }^{32}$ The phase of the reflectometry signal is inferred directly from the radial structure of the electric field, which is illustrated in Fig. 4 for both the O-mode (a) and X-mode (b) polarizations of the probing signal. The reflectometry signal as a function of time is then built by running the Helmholtz code for successive steps of the density fluctuations given by the GENE code (see Sec. II B). The phase fluctuations obtained for the $\mathrm{O}$-mode and $\mathrm{X}$-mode (the probing frequency being adjusted to probe the same radial position corresponding to the center of the fluctuation zone accordingly) in the LOC and the SOC regimes are depicted in Figs. 5(a)-5(d). Under the Born approximation valid for low levels of the density fluctuations, it was shown in previous works ${ }^{33-35}$ that the phase fluctuations could be retrieved directly from the integral of the density fluctuations along the radial line-of-sight

$$
\Delta \varphi \cong-2 \pi\left(k_{0} L\right)^{\frac{2}{3}} \int_{\xi_{\text {fluc }}} \frac{\delta n_{e}(\xi)}{n_{c}} A_{i}^{2}(\xi) h(\xi) d \xi,
$$

where $\xi$ represents the position normalized to a quantity related to the gradient length $L, k_{0}$ is the probing wavenumber in the vacuum, $n_{c}$ is the cut-off density, $A_{i}$ is the Airy function, and $h$ is a weighting function depending on the wave polarization. While $h$ equals to one for the O-mode, it can be expressed as follows for the $\mathrm{X}$-mode ${ }^{36}$

$$
h(\xi)=\frac{\left[\omega^{2}-2 \omega_{p e}^{2}(\xi)\right]\left[\omega^{2}-\omega_{c e}^{2}(\xi)\right]+\omega_{p e}^{4}(\xi)}{\left[\omega^{2}-\omega_{p e}^{2}(\xi)-\omega_{c e}^{2}(\xi)\right]^{2}},
$$

where $\omega, \omega_{p e}$, and $\omega_{c e}$ are the probing frequency, the electron plasma frequency, and the electron cyclotron frequency, respectively. Expression (2) indicates that (i) the amplitude of the phase fluctuations linearly depends on the level of density fluctuations and (ii) the main contribution comes from the cut-off layer vicinity where the Airy function is at its maximum value. As illustrated in Figs. 5(a)-5(d), a good agreement was found between the full-wave results and
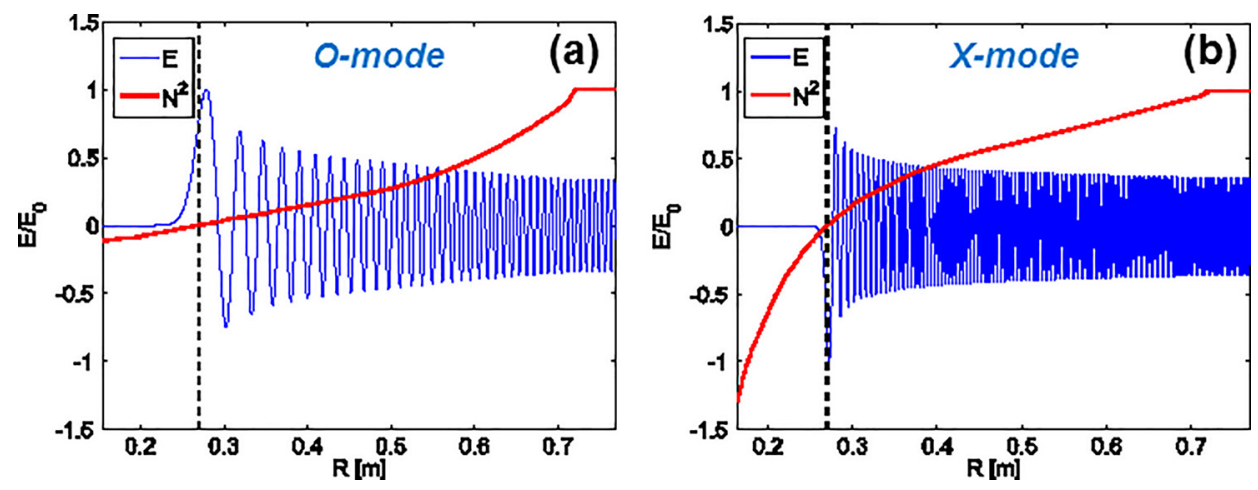

FIG. 4. Reflectometry simulation from the 1D Helmholtz code: example in the LOC case of the probing electric field computed for (a) the O-mode polarization and (b) the $\mathrm{X}$-mode polarization. As indicated in Fig. 3, (a) and (c) the probing frequency was adjusted (to $41 \mathrm{GHz}$ and $102 \mathrm{GHz}$ for the O-mode and $\mathrm{X}$-mode, respectively) in order to probe the center of the fluctuation region. 
LOC
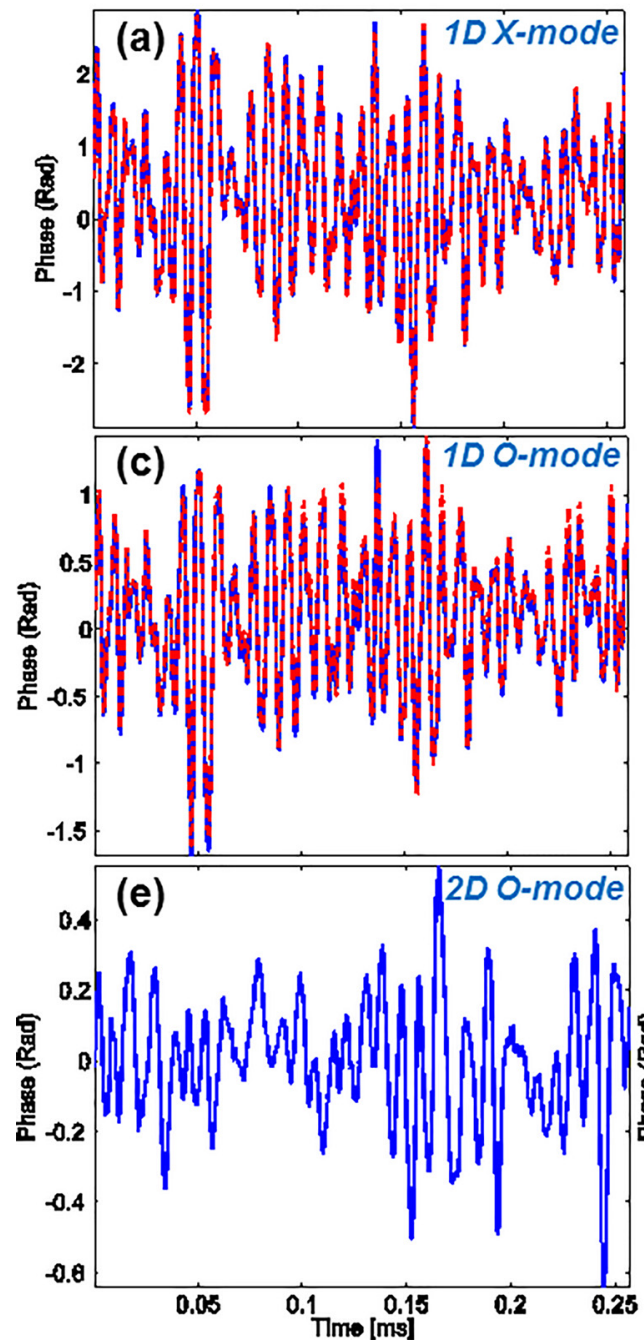

SOC
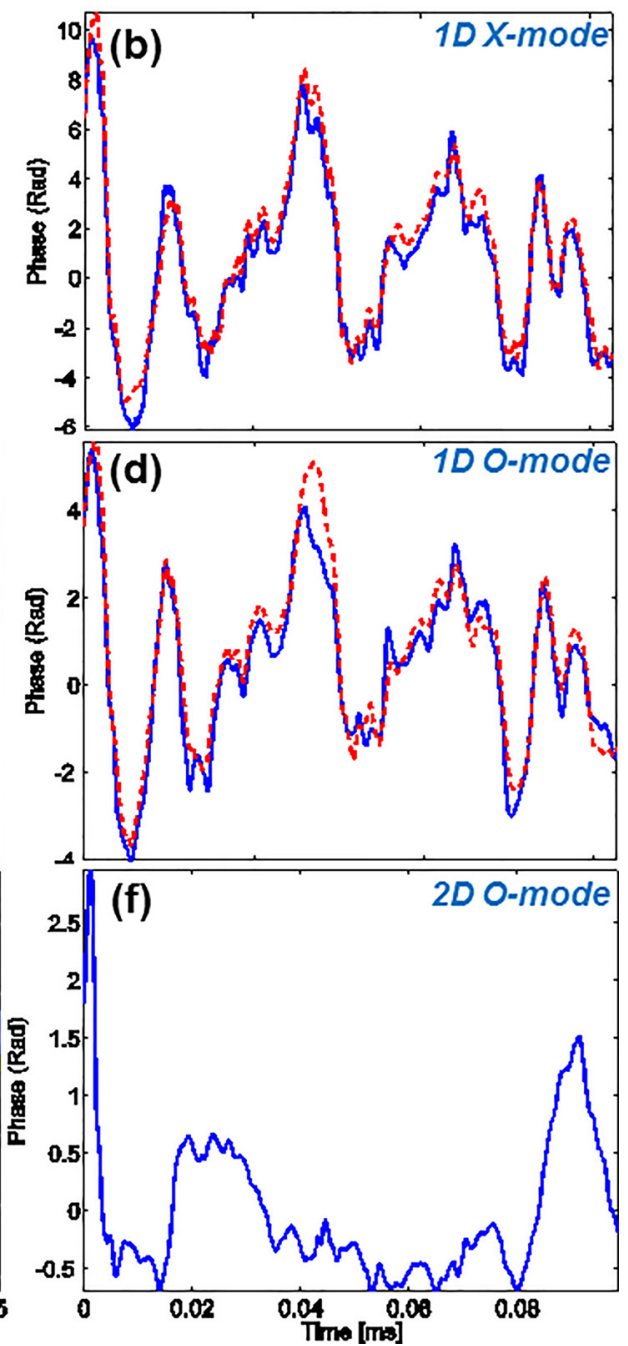

FIG. 5. Phase fluctuations in the LOC (left hand-side) and the SOC (right hand-side) regimes: (a) and (b) results from the 1D X-mode Helmholtz code (full lines) and analytical expression (dashed lines); (c) and (d) results from the 1D O-mode Helmholtz code (full lines) and analytical expression (dashed lines); and (e) and (f) results from the 2D O-mode FD-TD code. analytical expression (2), suggesting that the reflectometer response in the Tore Supra ohmic discharges (either in LOC or SOC regimes) satisfies the Born approximation.

A first comment regarding these results is that, although the amplitude of the phase fluctuations is larger for the $\mathrm{X}$-mode than for the O-mode (which can be explained by the fact that the wave-number $k_{0}$ and the weighting function $h$ in (2) are different), their pattern is identical whatever be the polarization mode. This is clearly observed in the spectra of the phase shown in Figs. 6(a) and 6(b) where both the $\mathrm{O}$-mode and the $\mathrm{X}$-mode results reproduce the same spectral shape. Moreover, one can notice that the level of phase fluctuations is larger in the SOC regime than in the LOC regime, in agreement with the larger level of the density fluctuations in the SOC regime $\left(\left\langle\delta \mathrm{n}_{e} / \mathrm{n}_{\mathrm{c}}\right\rangle \sim 0.45 \%\right)$ than in the LOC regime $\left(\left\langle\delta \mathrm{n}_{e} / \mathrm{n}_{\mathrm{c}}\right\rangle \sim 0.2 \%\right)$. It is also striking to note that, while a large broadband spectrum is observed in the SOC regime, a QC-TEM mode appears in the spectrum at around
LOC

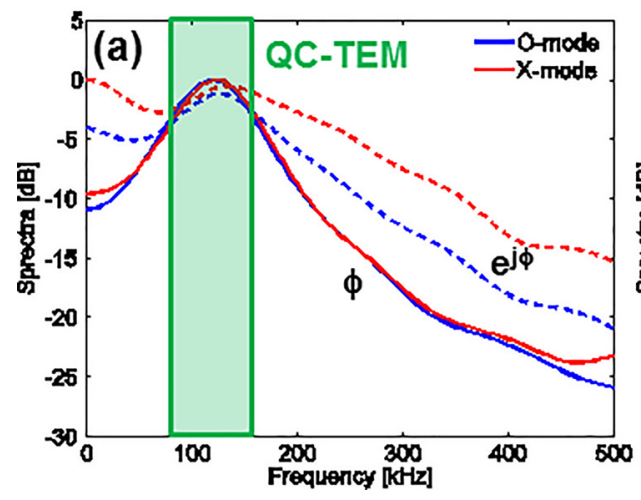

SOC

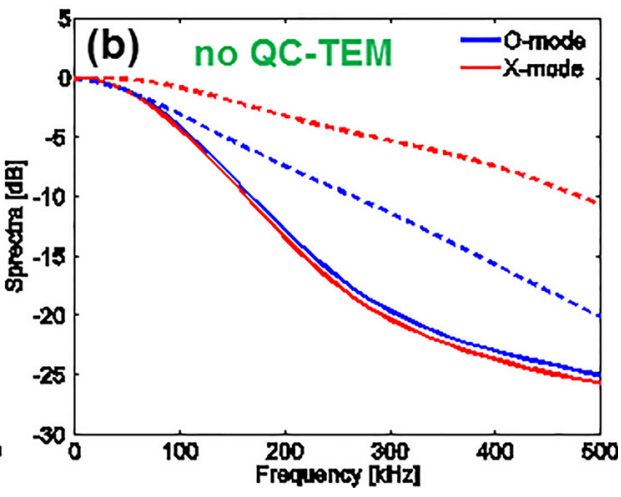

FIG. 6. A comparison of the 1D simulation results for the $\mathrm{O}$ and $\mathrm{X}$ mode polarizations: signal spectra in (a) the LOC regime and (b) the SOC regime. The full and the dashed lines represent the spectra of the phase $\phi$ and of the complex signal $\mathrm{e}^{\mathrm{j} \phi}$, respectively. 
$120 \mathrm{kHz}$ in the LOC regime, thereby confirming the experimental findings. ${ }^{10}$ The complex form a(t). $e^{j \varphi(t)}$ of the reflectometry signals instead of their phase is often used for spectral analysis, this having the effect to broaden the spectrum and add some harmonics resulting from a combination between the main frequency components. The spectra obtained from the FFT of the complex signals are also displayed (dashed lines) in Fig. 6. As expected, they appear wider than those obtained from the ones given by the FFT of the phase but the same behavior remains, i.e., a broadband spectrum is observed in the SOC regime while the QC mode is still detectable in the $\mathrm{LOC}$ regime.

\section{2D effects}

With the purpose to validate the $1 \mathrm{D}$ results presented in Sec. III B, 2D simulations were carried out with a 2D O-mode code using a 2 nd order FD-TD scheme. ${ }^{37,38}$ The code computes iteratively the probing electric field for a number of time steps large enough to ensure that the probing wave returns back to the receiving antenna. A transparent signal injection method ${ }^{39}$ allowing for a direct discrimination of the reflected wave is used to launch a Gaussian beam with similar characteristics than in the Tore Supra experiments. These computations are repeated for all the successive maps of the density fluctuations so that the synthetic reflectometry signal can be reconstituted. As shown in Figs. 5(e) and 5(f), similar to $1 \mathrm{D}$ simulation, the amplitude of the $2 \mathrm{D}$ phase fluctuations is larger in the SOC regime than in the LOC regime (consistently with the fact the level of turbulence is higher in the SOC regime). However, it was noted that the phase fluctuations have smaller amplitude in $2 \mathrm{D}$ simulations than in
1D simulations, whatever be the confinement regime. A likely explanation is that the $2 \mathrm{D}$ computations see the global movement of the density fluctuations, and subsequently, the apparent change of a refractive index is less substantial than in the 1D case. In Figs. 7(a) and 7(b) are compared the spectra of the complex signals obtained from the 1D and $2 \mathrm{D}$ simulations. An asymmetry of the spectra is noticeable (even from 1D simulations), resulting from the tilting of the turbulence structures given by GENE. Although the spectra appear to be wider from the 1D computations (in agreement with the larger level of the phase fluctuations), it is stressed that they qualitatively give the same results, in particular, the QC-TEM mode is well reproduced in the case of the LOC regime. Nonetheless, a discrepancy between the $1 \mathrm{D}$ and $2 \mathrm{D}$ results is found for the frequency of the QC-TEM mode $(\sim 120 \mathrm{kHz}$ vs. $\sim 90 \mathrm{kHz})$ as illustrated in Fig. 7(c). As the GENE simulations show that the various poloidal components of the density fluctuations can oscillate at slightly different frequencies, ${ }^{24}$ this discrepancy could be explained by the fact that the probing beam in the $2 \mathrm{D}$ simulations is sensitive to a larger range of poloidal wave-numbers than in the 1D simulations.

\section{Poloidal asymmetry}

Investigating the poloidal asymmetry of the density fluctuations is valuable for a better understanding of the turbulence processes but remains experimentally challenging as most of the core turbulence diagnostics usually probe the LFS equatorial mid-plane. The reflectometer diagnostics in TEXTOR $^{40}$ and $\mathrm{T}-10^{41}$ are equipped with the distinct sets of antennas installed in the mid-plane and the top regions and

\section{LOC}

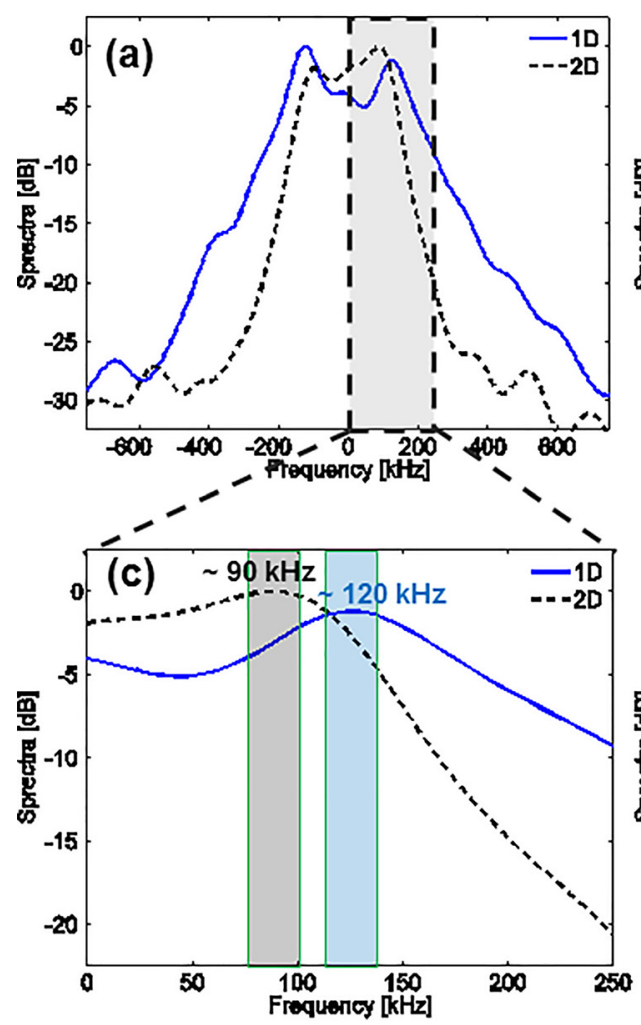

SOC

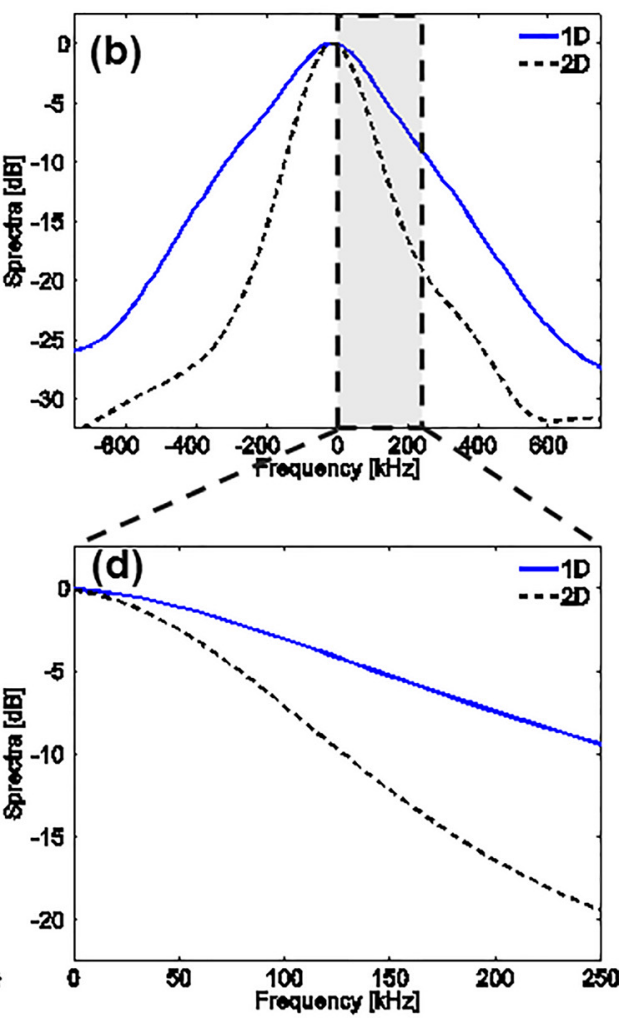

FIG. 7. A comparison of the $1 \mathrm{D}$ and 2D simulation results in the LOC (left hand-side) and the SOC (right handside) regimes: (a) and (b) signal spectra for O-mode and (c) and (d) zoom of (a) and (b). 
have provided some worthwhile indications on the turbulence poloidal asymmetry. It is quite straightforward to study this asymmetry with a synthetic reflectometry diagnostics. The reflectometer signal was then simulated for the density fluctuations given by the non-linear gyrokinetic data at each poloidal angle. The simulations were performed in an $1 \mathrm{D}$ geometry in order to keep the computing time reasonable. Although the 2D effects reported in Sec. III C were neglected, the results presented in Fig. 8 are expected to remain qualitatively pertinent. Figs. 8(a) and 8(b) show that the turbulence level is strongly ballooned in both the LOC and SOC regimes. A filtering around the QC-TEM mode frequency was also applied to the signal obtained in the LOC regime, demonstrating that the amplitude of the QC-TEM mode is a maximum in the low magnetic field side (LFS) region and decreases towards the high magnetic field side (HFS) region. Figs. 8(c) and 8(d) show the poloidal asymmetry of the fluctuation spectra, which look broader in the LFS region than in the top region, as usually noticed in the experiments. In the LOC regime, Fig. 8(c) shows a change of the QC-TEM mode frequency with respect to the poloidal position, as it may sometimes be observed in the experiments. ${ }^{42}$ In our case, no significant poloidal asymmetry of the turbulence phase velocity was found, and this change comes mainly from the poloidal asymmetry of the $\mathrm{E} \times \mathrm{B}$ rotation velocity. The poloidal asymmetry of the turbulence rotation velocity measured in experiments is still not fully understood though the impact of various factors excepted to contribute was discussed in a previous work. ${ }^{43}$

\section{DISCUSSION}

This paper reports on the simulation of reflectometry measurements in a Tore Supra Ohmic discharge, which highlights a modification of the turbulence spectrum in the LOC and SOC regimes. For a suitable turbulence model, the nonlinear gyrokinetic simulations from the GENE code were used as input in the full-wave reflectometry codes. First of all, it was found from the 1D full-wave computations that both O-mode and X-mode yield the same fluctuation spectra, even though the amplitude of phase fluctuations is larger for the X-mode. As presented in a previous paper ${ }^{10}$ and depicted in Figure 9, the synthetic reflectometry results are in a good agreement with the experimental observations. Outstandingly, a QC-TEM mode is recovered in the LOC regime only, at a frequency $(\sim 100 \mathrm{kHz})$ consistent with the experiments. It was also shown that the Born approximation valid for low levels of turbulence applies for a reflectometry measurement in the core region of the Tore Supra ohmic discharges. In the edge region of tokamak plasmas, the level of turbulence is usually high and previous studies ${ }^{44,45}$ have pointed out that microwave beams crossing such zones could be widened and distorted, with potential effects on the core reflectometry measurements and validity of the Born approximation. In this paper, one has not taken into account this effect, and a preliminary study of the impact of a high level of an edge turbulence on the core reflectometry measurement can be found in Ref. 46.

The $1 \mathrm{D}$ and $2 \mathrm{D}$ results were also compared in the $\mathrm{O}$ mode polarization case, which showed quite a good qualitative agreement. While the amplitude of phase fluctuations is smaller in the 2D case, the shape of fluctuation spectra is still pretty similar from both $1 \mathrm{D}$ and $2 \mathrm{D}$ calculations. However, a certain discrepancy for the QC-TEM mode frequency was noticed between the $1 \mathrm{D}$ results $(\sim 120 \mathrm{kHz})$ and the $2 \mathrm{D}$ results $(\sim 90 \mathrm{kHz})$. A probable explanation is that, in $2 \mathrm{D} \mathrm{sim}-$ ulations, the probing beam is more sensitive to different poloidal wavenumbers of turbulence than that in the 1D simulations. The non-linear gyrokinetic simulations suggest that the various poloidal components of the turbulence fluctuate
LOC

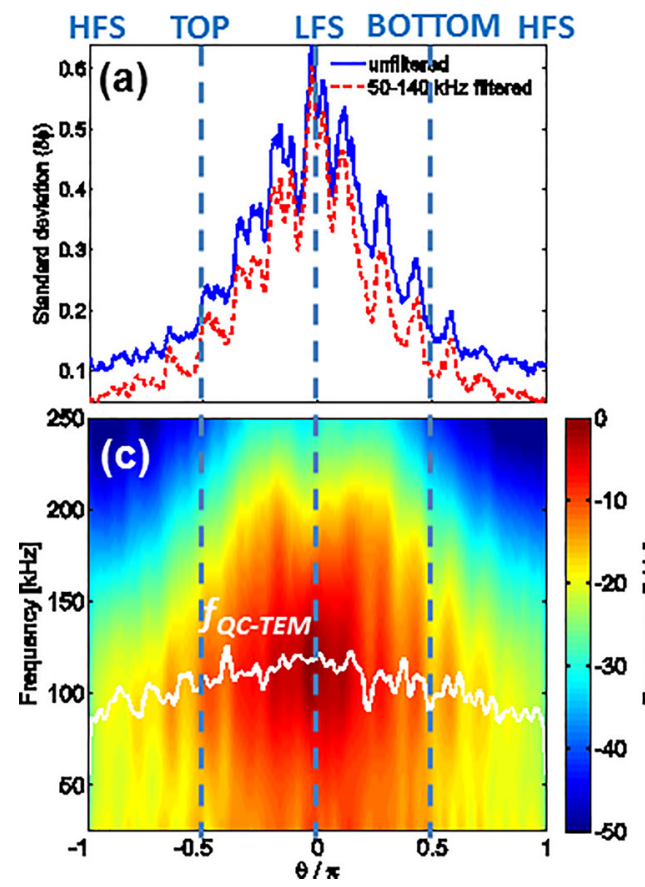

SOC

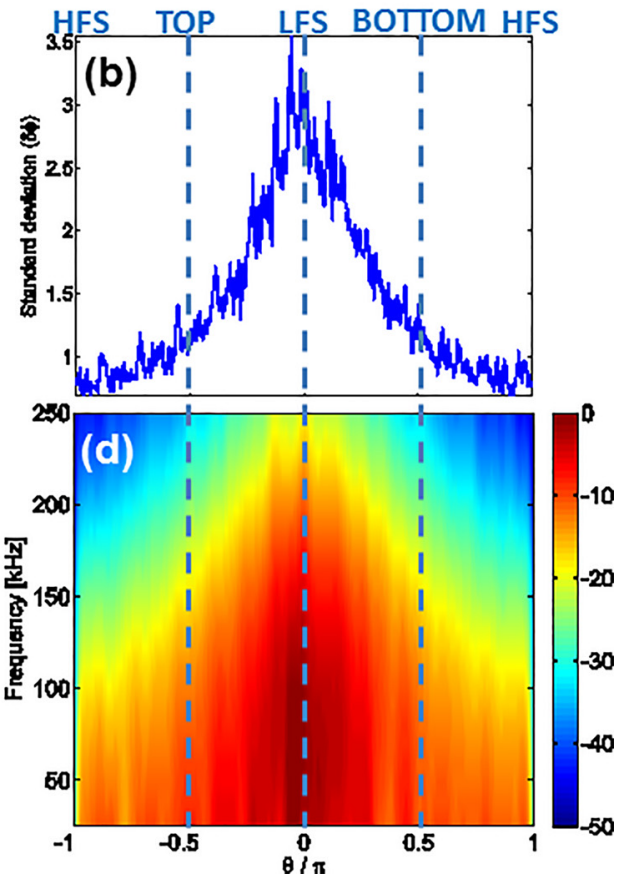

FIG. 8. The level of signal fluctuations in the LOC (left hand-side) and the SOC (right hand-side) regimes: (a) and (b) amplitude of phase fluctuations vs. the poloidal angle, (c) and (d) spectrogram of phase fluctuations vs. the poloidal angle. 
Simulation

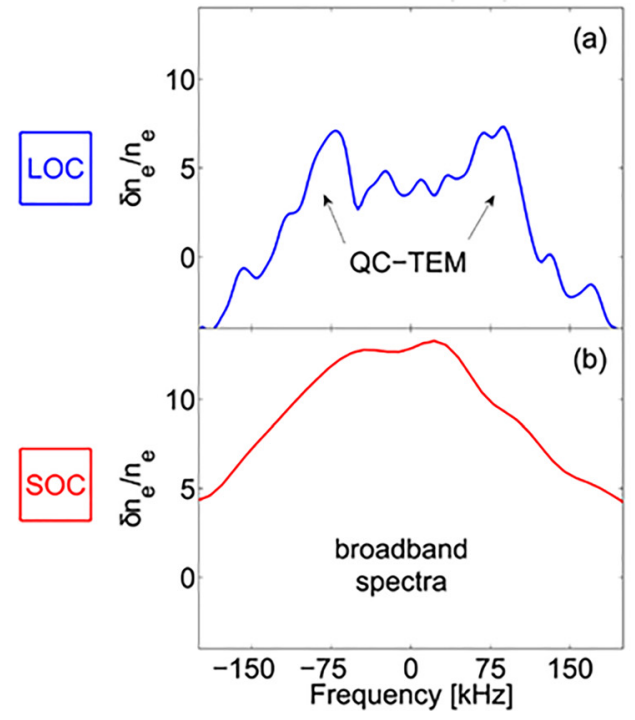

Experiment

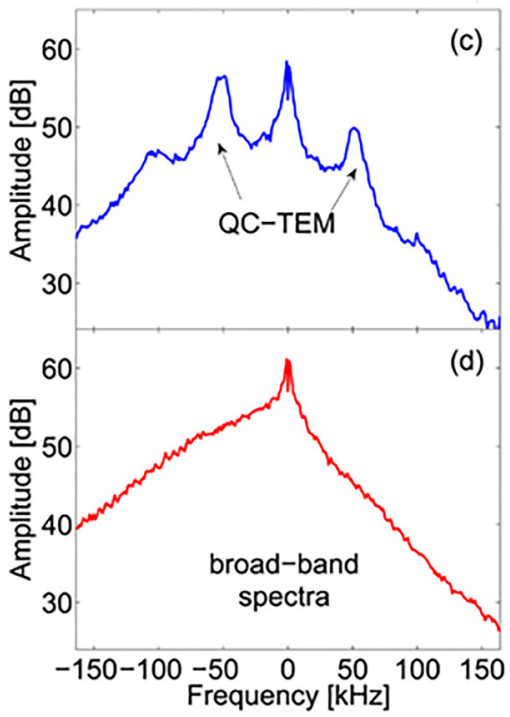

FIG. 9. A comparison between the synthetic reflectometry simulation (left hand-side) and experiment results (right hand-side) for the fluctuation spectra in the LOC and SOC regimes. Reprinted with permission from Arnichand et al., Plasma Phys. Controlled Fusion 58, 014037 (2016). Copyright 2016 EURATOM at slightly different frequencies, which could justify the divergence in the QC-TEM mode frequency deduced from the $1 \mathrm{D}$ and $2 \mathrm{D}$ results.

Finally, the poloidal asymmetry of the density fluctuations was investigated. First of all, a clear ballooning of the fluctuation level was found in a good agreement with experiments. In addition, a poloidal asymmetry of the QC-TEM frequency is observed in the LOC regimes. Such a change of QC mode frequency in the LFS and top regions is sometimes observed in the reflectometry measurements, but further investigations are still required to better understand its origin.

${ }^{1}$ C. P. Ritz, R. V. Bravenec, P. M. Schoch, R. D. Bengtson, J. A. Boedo, J. C. Forster, K. W. Gentle, Y. He, R. L. Hickok, Y. J. Ki et al., Phys. Rev. Lett. 62, 1844 (1989).

${ }^{2}$ X. Garbet, P. Mantica, C. Angioni, E. Asp, Y. Baranov, C. Bourdelle, R. Budny, F. Crisanti, G. Cordey, L. Garzotti et al., Plasma Phys. Control. Fusion 46, B557 (2004).

${ }^{3}$ J. E. Rice, I. Cziegler, P. H. Diamond, B. P. Duval, Y. A. Podpaly, M. L. Reinke, P. C. Ennever, M. J. Greenwald, J. W. Hughes, Y. Ma et al., Phys. Rev. Lett. 107, 265001 (2011).

${ }^{4}$ X. Garbet, L. Garzotti, P. Mantica, H. Nordman, M. Valovic, H. Weisen, and C. Angioni, Phys. Rev. Lett. 91, 035001 (2003).

${ }^{5}$ F. Jenko, D. Told, T. Görler, J. Citrin, A. Bañón Navarro, C. Bourdelle, S. Brunner, G. Conway, T. Dannert, H. Doerk et al., Nucl. Fusion 53, 073003 (2013).

${ }^{6}$ A. E. White, N. T. Howard, M. Greenwald, M. L. Reinke, C. Sung, S. Baek, M. Barnes, J. Candy, A. Dominguez, D. Ernst et al., Phys. Plasma 17, 020701 (2010).

${ }^{7}$ J. E. Rice, C. Gao, M. L. Reinke, P. H. Diamond, N. T. Howard, H. J. Sun, I. Cziegler, A. E. Hubbard, Y. A. Podpaly, W. L. Rowan et al., Nucl. Fusion 53, 033004 (2013).

${ }^{8}$ J. C. Hillesheim, J. C. DeBoo, W. A. Peebles, T. A. Carter, G. Wang, T. L. Rhodes, L. Schmitz, G. R. McKee, Z. Yan, G. M. Staebler et al., Phys. Rev. Lett. 110, 045003 (2013).

${ }^{9}$ J. S. Sarff, A. F. Almagri, J. K. Anderson, M. Borchardt, W. Cappechi, D. Carmody, K. Caspary, B. E. Chapman, D. J. Den Harlog, J. Duff et al., Nucl. Fusion 55, 104006 (2015).

${ }^{10}$ H. Arnichand, J. Citrin, S. Hacquin, R. Sabot, A. Krämer-Flecken, X. Garbet, C. Bourdelle, C. Bottereau, F. Clairet, J.-C. Giacalone et al., Plasma Phys. Controlled Fusion 58, 014037 (2016).

${ }^{11}$ R. Coelho, S. Äkäslompolo, A. Dinklage, A. Kus, R. Reimer, E. Sundén, S. Conroy, E. Blanco, G. Conway, S. Hacquin et al., Fusion Sci. Technol. 63(1), 1 (2013).
${ }^{12}$ S. Heuraux, E. Faudot, F. da Silva, J. Jacquot, L. Colas, S. Hacquin, N. Teplova, K. Syseova, and E. Gusakov, C. R. Phys. 15(5), 421 (2014).

${ }^{13}$ H. Arnichand, R. Sabot, S. Hacquin, A. Krämer-Flecken, X. Garbet, J. Citrin, C. Bourdelle, G. Hornung, J. Bernardo, C. Bottereau et al., Nucl. Fusion 54, 123017 (2014).

${ }^{14}$ G. D. Conway, Nucl. Fusion 46, S799 (2006).

${ }^{15}$ C. Angioni, R. M. McDermott, F. J. Casson, E. Fable, A. Bottino, R. Dux, R. Fischer, Y. Podoba, T. Pütterich, F. Ryter et al., Phys. Rev. Lett. 107, 215003 (2011)

${ }^{16}$ C. Gao, J. E. Rice, H. J. Sun, M. L. Reinke, N. T. Howard, D. Mikkelson, A. E. Hubbard, M. A. Chilenski, J. R. Walk, J. W. Hughes et al., Nucl. Fusion 54, 083025 (2014).

${ }^{17}$ R. Sabot, A. Sirinelli, J.-M. Chareau, and J.-C. Giacalone, Nucl. Fusion 46, S685 (2006).

${ }^{18}$ V. A. Vershkov, D. A. Shelukhin, S. V. Soldatov, A. O. Urazbaev, S. A. Grashin, L. G. Eliseev, A. V. Melnikov, and the T-10 team, Nucl. Fusion 45, S203 (2005).

${ }^{19}$ A. Krämer-Flecken, V. Drevat, S. Soldatov, A. Rogister, V. Vershkov, and the TEXTOR team, Nucl. Fusion 44, 1143 (2004).

${ }^{20}$ H. Arnichand, R. Sabot, S. Hacquin, A. Krämer-Flecken, C. Bourdelle, J. Citrin, X. Garbet, J.-C. Giacalone, R. Guirlet, J. C. Hillesheim et al., Nucl. Fusion 55, 093021 (2015).

${ }^{21}$ V. A. Vershkov, V. F. Andreev, A. A. Borschegovskiy, V. V. Chistyakov, M. M. Drevin, L. G. Eliseev, E. P. Gorbunov, S. A. Grashin, A. V. Khmara, A. Y. Kislov et al., Nucl. Fusion 51, 094019 (2011).

${ }^{22}$ A. Krämer-Flecken, S. Soldatov, Y. Xu, H. Arnichand, S. Hacquin, R. Sabot, and the TEXTOR team, New J. Phys. 17, 073007 (2015).

${ }^{23}$ F. Jenko, Phys. Plasma 7, 1904 (2000).

${ }^{24}$ J. Citrin, H. Arnichand, J. Bernardo, C. Bourdelle, X. Garbet, S. Hacquin, and R. Sabot, US-EU TTF Conference, Culham (2014).

${ }^{25}$ JF. Artaud, V. Basiuk, F. Imbeaux, M. Schneider, J. Garcia, G. Giruzzi, P. Huynh, T. Aniel, F. Albajar, J.-M. Ane et al., Nucl. Fusion 50(4), 043001 (2010)

${ }^{26}$ D. G. Swanson, Plasma Waves, 2nd ed. (IOP Publishing Ltd., 2003).

${ }^{27}$ E. Mazzucato, Phys. Fluids B 4, 3460 (1992).

${ }^{28}$ R. M. McDermott, C. Angioni, G. D. Conway, R. Dux, E. Fable, R. Fischer, T. Pütterich, F. Ryter, E. Viezzer, and the ASDEX Upgrade team, Nucl. Fusion 54, 043009 (2014).

${ }^{29}$ E. Trier, L.-G. Eriksson, P. Hennequin, C. Fenzi, C. Bourdelle, G. Falchetto, X. Garbet, T. Aniel, F. Clairet, and R. Sabot, Nucl. Fusion 48, 092001 (2008).

${ }^{30}$ S. Hacquin, S. Heuraux, M. Colin, and G. Leclert, J. Comput. Phys. 174(1), 1 (2001)

${ }^{31}$ G. Zadvitskiy, S. Heuraux, C. Lechte, S. Hacquin, and R. Sabot, IRW12 Workshop, Jülich (2015).

${ }^{32} \mathrm{~A}$. Taflove and S. C. Hagness, Computational Electrodynamics, 2nd ed. (Artech House, 2000).

${ }^{33}$ X. L. Zou, L. Laurent, and J. M. Rax, Plasma Phys. Controlled Fusion 33(8), 903 (1991). 
${ }^{34}$ I. H. Hutchinson, Plasma Phys. Controlled Fusion 34(7), 1225 (1992).

${ }^{35}$ C. Fanack, I. Boucher, F. Clairet, S. Heuraux, G. Leclert, and X. L. Zou, Plasma Phys. Controlled Fusion 38, 1915 (1996).

${ }^{36}$ E. Z. Gusakov and A. Y. Popov, Plasma Phys. Controlled Fusion 46, 1393 (2004).

${ }^{37}$ B. I. Cohen, B. B. Afeyan, A. E. Chou, and N. C. Luhmann, Jr., Plasma Phys. Controlled Fusion 37, 329 (1995).

${ }^{38}$ S. Hacquin, H. Arnichand, G. Dif-Pradalier, S. Heuraux, R. Sabot, Y. Sarazin, and the Tore Supra team, IRW11 Workshop, Palaiseau (2013).

${ }^{39}$ F. da Silva, S. Heuraux, S. Hacquin, and M. E. Manso, J. Comput. Phys. 203, 467 (2005).

${ }^{40}$ A. Krämer-Flecken, S. Soldatov, B. Vowinkel, and P. Müller, Rev. Sci. Instrum. 81, 113502 (2010).
${ }^{41}$ V. A. Vershkov, V. V. Dreval, and S. V. Soldatov, Rev. Sci. Instrum. 70, 1700 (1999).

${ }^{42}$ A. Krämer-Flecken, private communication (2015).

${ }^{43}$ A. Storelli, L. Vermare, P. Hennequin, Ö. D. Gürcan, G. Dif-Pradalier, Y. Sarazin, X. Garbet, T. Görler, R. Singh, P. Morel et al., Phys. Plasma 22, 062508 (2015).

${ }^{44}$ E. Z. Gusakov and A. Yu. Popov, Plasma Phys. Controlled Fusion 44, 2327 (2002).

${ }^{45}$ E. V. Sysoeva, F. da Silva, E. Z. Gusakov, S. Heuraux, and A. Yu Popov, Nucl. Fusion 55, 033016 (2015).

${ }^{46}$ G. Zadvitskiy, S. Heuraux, C. Lechte, S. Hacquin, R. Saot, and F. Clairet, in Proceedings of the 43rd EPS Conference on Plasma Physics, Leuven, Belgium, 4-8 July 2016. 